\title{
Prenatal ultrasound diagnosis and management of fetal aortopulmonary septal defects: a case series
}

\author{
Junjian Yu, Kang Liu, Weichang Xu, Jianxian Xiong, Xuehong Zhong, Shuo Hu, Wentong Li \\ Department of Cardiovascular and Thoracis Surgery, The First Affiliated Hospital of Gannan Medical University, Ganzhou, China \\ Contributions: (I) Conception and design: J Yu, W Li; (II) Administrative support: W Li; (III) Provision of study materials or patients: W Xu; (IV) \\ Collection and assembly of data: J Xiong, X Zhong; (V) Data analysis and interpretation: S Hu; (VI) Manuscript writing: All authors; (VII) Final \\ approval of manuscript: All authors. \\ Correspondence to: Wentong Li. Department of Cardiovascular and Thoracis Surgery, The First Affiliated Hospital of Gannan Medical University, \\ Ganzhou, China. Email: Tongge87821@sina.com.
}

\begin{abstract}
Background: To investigate the prenatal ultrasound diagnosis and management of patients with aortopulmonary septal defects (APSDs).

Methods: A total of 8 fetuses with APSDs who underwent fetal echocardiography at our hospital from January 2015 to January 2019 were retrospectively included in this study.

Results: Among the 8 fetuses, there were 4 cases of type I APSD, 3 cases were type II, and 1 case was type III. Among the 8 cases, there were 2 cases of simple APSD. There were echocardiographic characteristics that were common to all 3 types of APSD. This included defects between the ascending aorta and the trunk of the pulmonary artery in the short-axis section of the aorta, and in the three vessels and the three-vessel trachea section. Furthermore, the "V"-shaped structure confluence point of all APSD cases was positioned more forward than normal in the three-vessel trachea section. Type I APSD can be better characterized by the cross-section of the double outflow tract of the aorta and the pulmonary artery, which is close to the aortic valve and pulmonary valve. Type II APSD can be clearly diagnosed by the short-axis view of the aorta. Since the defect between the aorta and the pulmonary artery is distant from the aortic valve and pulmonary valve, the defect does not involve the bifurcation of the pulmonary artery and may be associated with an ectopic origin of the right pulmonary artery. Type III APSD is similar to a permanent arterial trunk, and the space between the ascending aorta and the trunk of the pulmonary artery is completely missing. Color and pulse Doppler showed shunt flow in the defects.
\end{abstract}

Conclusions: APSD can be diagnosed and classified by fetal echocardiography. This, together with the presence or absence of fetal intracardiac and extracardiac deformities, can provide valuable prenatal information to pregnant women and their families, which may facilitate timely diagnosis and timely surgical treatment after birth.

Keywords: Prenatal ultrasound; aortopulmonary septal defect (APSD); classification

Submitted Sep 09, 2021. Accepted for publication Nov 16, 2021.

doi: $10.21037 / \mathrm{tp}-21-488$

View this article at: https://dx.doi.org/10.21037/tp-21-488

\section{Introduction}

Aortopulmonary septal defect (APSD), also known as an aortopulmonary window (APW), is a congenital heart disease involving a direct communication between the ascending aorta and the pulmonary trunk, even though the aortic and pulmonary valves develop normally. This condition is rare, accounting for $0.10-0.60 \%$ of congenital heart defects (1). After birth, neonates with APSD present with large amounts of regurgitation between the aorta and pulmonary artery. The symptoms are severe and appear early, and the disease progresses rapidly. APSD is often 
complicated with heart failure or pulmonary infection, and the prognosis is poor. Timely diagnosis and treatment is crucial to minimizing perinatal mortality and improving patient prognosis. With the widespread application of prenatal echocardiography in hospitals at all levels in China, the detection rate of APSD is increasing and there are increasing reports regarding the prenatal diagnosis of main pulmonary septal defects $(2,3)$. However, due to the low incidence of the disease, there is still a lack of experience and understanding of APSD, and missed diagnosis can lead to serious consequences. Therefore, to improve the prenatal diagnosis and management of this condition, ultrasonographic images of 8 fetuses with APSD were retrospectively analyzed. The image characteristics were summarized and their relationship with patient outcomes was reported. We present the following article in accordance with the AME Case Series reporting checklist (available at https://dx.doi.org/10.21037/tp-21-488).

\section{Methods}

\section{Research objective}

A total of 8 fetuses with APSD confirmed by postpartum echocardiography, postpartum echocardiography, autopsy, cardiac catheterization or surgery from January 2015 to January 2019 were analyzed retrospectively. The average age of the pregnant women was $27.9 \pm 4.3$ years (range, 26-34 years) and the average gestational age as diagnosed by prenatal ultrasound was $26.2 \pm 3.7$ weeks (range, $19.5-$ 35.0 weeks). The fetuses were all singletons. In this group of 8 cases, six cases were first births, two cases were second births. All the parents and their first child had normal hearts.

\section{Instruments and methods}

The study was conducted in accordance with the Declaration of Helsinki (as revised in 2013). The study was approved by Scientific Research Ethics Committee of the First Affiliated Hospital of Gannan Medical College (No. LLSC-202105401) and informed consent was taken from all the patients' guardians.

\section{Instruments}

The GEVolusonE8 and Samsung WS80A color Doppler ultrasound machines were used with a two-dimensional convex array probe at a frequency of $2-7 \mathrm{MHz}$.

\section{Inspection method}

The fetal heart mode was selected on the imaging machine and the sequential segmented analysis method was used to examine the fetal heart. First, the fetal position was determined, the position of the heart was judged, the horizontal cross-section of the gastric vesicle in the upper abdomen of the fetus was obtained, and then the positional relationship between the fetal heart and the viscera was determined. The following 5 standard sections of the fetal heart were displayed sequentially: fetal fourchamber section, fetal five-chamber section, basal short axis and the pulmonary artery bifurcation section, fetal left ventricular outflow tract and the aortic arch long axis section, and the fetal right ventricular outflow tract and the long axis section of the ductus arteriosus. The crosssection from the top to bottom of the upper chest of the fetus was then scanned continuously to show the threevessel section, the three-vessel trachea section ( $3 \mathrm{VT})$, and the three-vessel pulmonary artery branch section. On the basis of these sections, color Doppler and energy Doppler flow imaging were added to observe the blood flow in the atrioventricular cavity, the valves, and the large vessels. For patients suspected of having APSD, the short axis of the great arteries, the three vessels, the 3 VT section, and the coronary section of the double outflow tract of the aorta were scanned.

\section{Typing}

Richardson et al. (4) divided APSD into 3 types according to the mechanism of embryonic development and the location of the disease. Type I is proximal APSD where the defect is located in the wall of the ascending aorta and above the Valsalva sinus. Type II is a distal APSD where the defect is located between the distal end of the ascending aorta and the pulmonary artery bifurcation, often associated with an ectopic origin of the right pulmonary artery. Type III is a primary complete pulmonary septal defect, where the abnormal right pulmonary artery originates from the ascending aorta.

\section{Follow-up and verification}

The fetal examinations and diagnoses were performed jointly by 2 senior physicians. After delivery, the neonates were taken to the Children's Hospital for surgical treatment. Complete data were obtained by telephone or WeChat follow-up. 

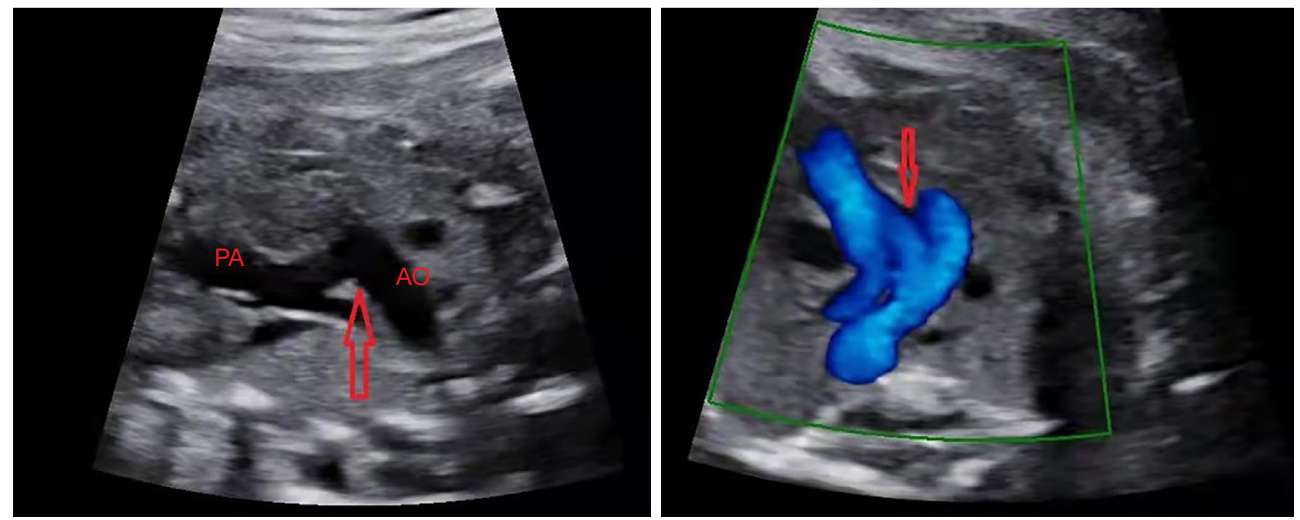

Figure 1 Representative ultrasound images of a type I aortopulmonary septal defect case in utero. APSD represents the main pulmonary artery window; red upward arrow indicates the main pulmonary septal defect; red downward arrow indicates the shunt, main pulmonary septal defect type I, located proximal to the aortic valve. PA, pulmonary artery; AO, aorta; APSD, aortopulmonary septal defect.
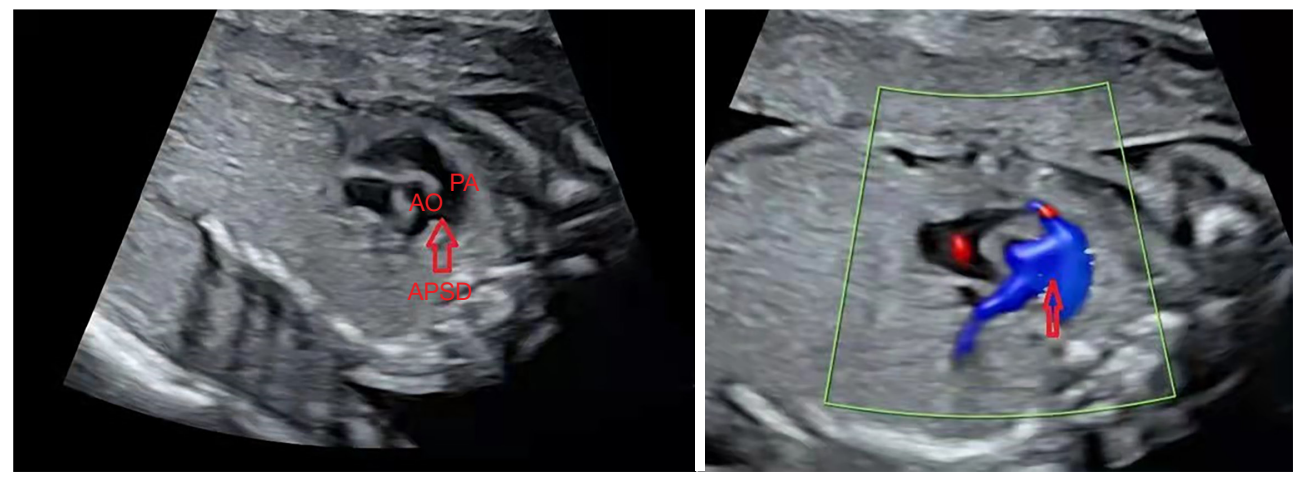

Figure 2 Representative ultrasound images of a type II aortopulmonary septal defect case in utero. APSD represents the main pulmonary artery window; red upward arrow indicates the main pulmonary septal defect and the shunt between the right pulmonary artery and the aorta. The defect is located between the right pulmonary artery and the aorta, away from the pulmonary valve. PA, pulmonary artery; AO, aorta; APSD, aortopulmonary septal defect.

\section{Results}

Among the 8 cases of APSD, there were 4 cases of type I APSD (Figure 1), 3 cases of type II (between the posterior wall of the aorta and the beginning of the right pulmonary artery; Figure 2), and 1 case of type III. Among the 8 fetuses, there were 2 cases of simple APSD, 1 case of APSD with a persistent left superior vena cava, and 1 case of Berry syndrome (type II aorta-pulmonary septal defect, right pulmonary artery originating from the aorta, coarctation or amputation of the aortic arch).

All neonates recovered well after surgery. There was 1 case of Berry syndrome complicated with anomalous pulmonary venous drainage, 2 cases with intracardiac multiple abnormalities $(1$ case with tetralogy of Fallot and pulmonary atresia, and 1 case with single atrium, single ventricle, and aortic valve atresia), and 1 case with intracardiac multiple abnormalities and extracardiac abnormalities (including ventricular septal defect, right aortic arch, and hydrocephalus). See Table 1 for details.

All 8 types of fetal APSD showed common ultrasound characteristics. The cross-section of the double outflow tract of the aorta and pulmonary artery, the short axis section of the great artery, and the three-vessel and the three-vessel trachea section all showed absence of a septal echo between the pulmonary artery and the ascending aorta. Furthermore, the confluence point of the " $\mathrm{V}$ " structure of the three-vessel trachea section was higher than that of the normal ductus arteriosus (Figure 3). In addition, color 
Table 1 Prenatal ultrasonic classification, postpartum condition, and outcome of 8 fetuses with main pulmonary septal defects

\begin{tabular}{|c|c|c|c|c|}
\hline $\begin{array}{l}\text { Number } \\
\text { of cases }\end{array}$ & $\begin{array}{l}\text { Gestational week at } \\
\text { examination }\left(\text { week }^{+d}\right)\end{array}$ & $\begin{array}{l}\text { Prenatal complicated with intracardiac and } \\
\text { extracardiac malformations }\end{array}$ & $\begin{array}{l}\text { Prenatal } \\
\text { ultrasonic typing }\end{array}$ & Result \\
\hline 1 & $24^{+2}$ & APSD, tetralogy of Fallot with pulmonary atresia & Type I & Confirmation of induced labor \\
\hline 3 & $19^{+5}$ & $\begin{array}{l}\text { Single atrium, single ventricle, aortic valve atresia, } \\
\text { APSD }\end{array}$ & Type I & Confirmation of induced labor \\
\hline 5 & $25^{+3}$ & Berry syndrome & Type II & Confirmed by postnatal surgery \\
\hline 6 & $24^{+5}$ & $\begin{array}{c}\text { Berry syndrome, anomalous pulmonary venous } \\
\text { drainage }\end{array}$ & Type II & Confirmation of induced labor \\
\hline 7 & $26^{+5}$ & APSD & Type II & Confirmed by postnatal surgery \\
\hline
\end{tabular}

APSD, aortopulmonary septal defect.

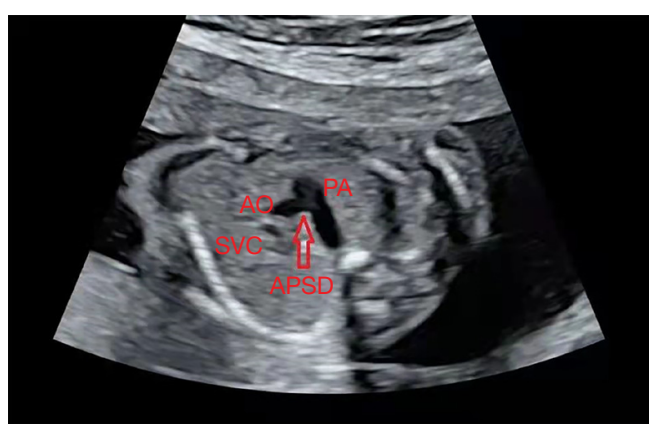

Figure 3 A representative ultrasound image showing the common characteristics in all 3 types of fetal aortopulmonary septal defect. APSD represents the main pulmonary artery window; red upward arrow indicates the main pulmonary septal defect, and the confluence point of the $\mathrm{V}$-shaped structure of the three-vessel trachea section is forward from normal. PA, pulmonary artery; AO, aorta; SVC, superior vena cava; APSD, aortopulmonary septal defect.

Doppler showed echo loss (interruption) and a left-to-right shunt signal.

There were specific ultrasonographic manifestations of the various types of APSD in the fetal period. Type I APSD (proximal type) showed interrupted echo of the proximal septum between the ascending aorta and the pulmonary trunk, close to the aortic valve and the pulmonary valve, and the cross-section of the double outflow tract of the aorta and pulmonary artery was easily observed. Type II APSD (distal type) showed interruption of the echoes at the bifurcation between the left posterior wall of the ascending aorta and the main pulmonary artery, often close to the right pulmonary artery, away from the aortic valve and the pulmonary valve, and was associated with an ectopic origin of the right pulmonary artery. The short axis section of the large arteries was easily observed. Type III APSD showed a complete absence of a septum between the aorta and the pulmonary artery, similar to persistent truncus arteriosus, and the three-vessel and three-vessel tracheal sections were easily observed.

\section{Discussion}

The primary purpose of fetal echocardiography is to maximize the detection of cardiac malformations in the second trimester. It can be applied to the evaluation of low-risk fetuses. It also helps identify the risk of fetal genetic syndrome, and can provides clinical materials for prepartum consultation, obstetrics management and multicenter therapy. There are two main reasons why a fetus needs a fetal echocardiogram. The first is the pregnant mother's factor, for example, pregnant mothers have diabetes, autoimmune disease, and there was an infection early in the pregnancy. Another factor is caused by the fetus, Abnormalities of cardiac structure, arrhythmias, or extra-cardiac structure were found during the ultrasound examination of fetus.

APSD is manifested as an incomplete separation of the fetal arteries into the aorta and pulmonary artery, leaving 
defects between the ascending aorta and the pulmonary artery. It is a rare congenital cardiovascular malformation, accounting for $0.1-0.6 \%$ of all congenital heart diseases. After birth, due to the defect between the aorta and the pulmonary artery, the significant left-to-right shunt can often lead to heart failure, and thus, this requires early surgical treatment. Therefore, efficient and accurate prenatal diagnosis of APSD is crucial for improving pregnancy outcomes, especially in relation to selecting the optimal neonatal delivery mode and performing timely operations (4-6).

Type I APSD is common, while types II and III are rare. In this study cohort, there were 4 cases of type I APSD, 3 cases of type II, and 1 case of type III. It is noteworthy that the proportion of type II APSD in this study was $3 / 8$, which is different from that reported in the literature and is likely due to the small sample size of the current investigation.

The three types of APSD showed common manifestations on prenatal ultrasound. The cross-section of the double outflow tract of the aorta and pulmonary artery, the short axis section of the great artery, and the three vessels and three vessels trachea section showed the absence of a septal echo between the pulmonary artery and ascending aorta. The confluence point of the "V" structure of the three-vessel trachea section was higher than that of the normal ductus arteriosus. Furthermore, the color Doppler showed echo loss (interruption) and a left-toright shunt signal. The fetal acoustic window was abundant and not disturbed by that of the lung. False loss could be avoided by adjusting the section, making APSD easier to diagnose by prenatal ultrasound than after birth. The threevessel section was the optimal section for the diagnosis of APSD as it could be obtained more easily in the fetus than in the neonate and it clearly showed the absence of a septal echo between the pulmonary artery and the ascending aorta. Due to the existence of the foramen ovale and ductus arteriosus in the fetus, the hemodynamics of APSD are not consistent with those postnatally. Enlargement of the left heart is not obvious when APSD occurs, and this mainly depends on direct signs. Therefore, attention should be given to the observation of the three-vessel and the threevessel tracheal sections, cross sections of the double outflow tract of the aorta and pulmonary artery, short-axis sections of the great arteries, and the color Doppler.

There were common manifestations that were specific to different types of APSD. Type I (proximal type) showed interruption of the septum between the ascending aorta and the pulmonary trunk, which is close to the aortic valve and pulmonary valve. Type II (distal type) showed interruption of the septum between the left posterior wall of the ascending aorta and the bifurcation of the main pulmonary artery, often close to the right pulmonary artery, far away from the aortic valve and pulmonary valve, and this may be associated with the ectopic origin of the right pulmonary artery. Type III showed complete absence of the septum between the aorta and pulmonary artery, similar to persistent truncus arteriosus. Since the 3 types of APSD show defects in different locations, the sections that are characteristic of each type are also different. For type I (proximal type), the cross-section of the double outflow tract of the aorta and the pulmonary artery, the short axis section of the large artery, and the three-vessel and threevessel trachea section were easily observed. Therefore, for the diagnosis and typing of APSD, particular attention should be focused on the scan of the above-mentioned sections.

When diagnosing APSD, attention should be given to the exclusion of Berry syndrome. In this study, 8 cases of type II APSD were complicated with Berry syndrome. Berry syndrome was first reported by Berry in 1982, including type II APSD, hypoplasia or amputation of the aortic arch, the origin of the ascending aorta from the right pulmonary artery, patent ductus arteriosus and intact interventricular septum. The incidence is very low, accounting for approximately $0.046 \%$ of patients with congenital heart disease (7). The main pulmonary septal defect of fetal Berry syndrome is often mistaken for ductus arteriosus, which leads to a missed diagnosis of aortic arch amputation and the main pulmonary septal defect. The three-vessel section combined with the aortic arch section shows that the threevessel section of the main pulmonary septal defect does not form a normal "V" type, and the confluence point is higher than the "V" type formed by the normal ductus arteriosus. Therefore, when diagnosing APSD, we must take note of the abnormal origin of the right pulmonary artery and the abnormality of the aortic arch and be attentive to the scanning of the three-vessel section combined with the aortic arch section.

APSD is easily complicated by other cardiovascular malformations, such as ventricular septal defects, aortic arch stenosis, and tetralogy of Fallot $(1,4,5)$. Among the 8 cases in this study, only 2 cases were simple APSD, and the other 6 cases were complicated with cardiovascular malformations. It was previously reported (7) that APSD is most often associated with amputation of the aortic arch, followed by patent ductus arteriosus, ventricular septal defect, coronary 
artery defect, and tetralogy of Fallot. In agreement with Luo et al. (8), the most common abnormality identified in this current investigation was an abnormality of the aortic arch.

Therefore, prenatal ultrasound can be used to effectively detect and diagnose APSD, with particular focus on the examination of other cardiac structures so as to exclude other structural abnormalities. It should be noted that cardiac malformations such as ventricular septal defects, aortic arch stenosis, and tetralogy of Fallot are more common than APSD. Thus, when ventricular septal defects and other malformations are identified, it is crucial to differentiate between APSD and other cardiac malformations.

Fetal APSD should be differentiated from persistent truncus arteriosus and doubly committed subarterial ventricular septal defect, Persistent truncus arteriosus shows only one group of semilunar valves, and APSD shows two groups of semilunar valves. The main difference between persistent truncus arteriosus and doubly committed subarterial ventricular septal defect is their position, The former is located above the pulmonary valve, the latter is located below the pulmonary valve.

As neonates with APSD are prone to early cardiac insufficiency and pulmonary hypertension, timely surgical intervention is crucial. It has been reported in the literature that APSDA is the most commonly used surgical method for repairing defects via the aorta (9). In this study cohort, there were 4 cases of type I APSD, included 2 cases of simple APSD, 1 case with a persistent left superior vena cava, and 1 case of Berry syndrome. All surgical operations were successful. The postoperative follow-up was satisfactory and there were no reported complications, which was consistent with the good prognosis of single cases of APSD reported in the literature (10). Due to the large gap in patients with APSD, there was a large number of left-to-right shunts, resulting in severe pulmonary hypertension in the neonatal period. If not treated in time, these children will likely have pulmonary hypertension and may die of heart failure or severe pneumonia. Therefore, early diagnosis and early treatment is the key to a good prognosis (11). Simple APSD can be detected and diagnosed accurately before delivery and immediately operated on after birth, resulting in a good prognosis.

None of these 8 cases of ASPD underwent chromosome examination. Previous studies have reported no significant correlation between single APSD and 22q11 microdeletion (12), and the probability of an overt chromosome abnormality is thus relatively low (2). However, the genetic correlation between Berry syndrome and APSD is relatively high. Remon et al. (13) reported that Berry syndrome was frequently complicated with trisomy 13. In this report, all 8 pregnant women refused to undergo chromosome karyotype examination, hence, the correlation between these cases and chromosomal abnormalities remains unknown.

However, echocardiography is the preferred imaging examination for fetal congenital heart disease, the result of echocardiography diagnosis is greatly influenced by ultrasonologist, and to check the gestational age requirements are more stringent. The development of MRI technology has gradually been applied to the diagnosis of fetal congenital abnormalities. The aorta can be clearly displayed, compared with echocardiography, it can better show the relationship between the position of the heart's great vessels and surrounding organs. It compensates for the shortcomings of the echocardiogram.

Echocardiography combined with MR can effectively improve the detection rate and accuracy of fetal congenital heart disease.

In summary, prenatal ultrasound can accurately diagnose the location, size, classification, and other features of APSD defects and it is currently the most commonly used method for prenatal examination. When prenatal APSD is detected, we should pay particular attention to assessing whether it is complicated with Berry syndrome, consider chromosome karyotype analysis, and transfer the neonate to a corresponding children's hospital or surgical hospital shortly after birth to facilitate timely surgical repair. The sample size of patients in this study is too small, so we need to increase the sample size for verification.

\section{Acknowledgments}

Funding: This study was supported by the Education Department Science and Technology Research Project of Jiangxi Provincial (Youth Project) (190821).

\section{Footnote}

Reporting Checklist: The authors have completed the AME Case Series reporting checklist. Available at https://dx.doi. org/10.21037/tp-21-488

Data Sharing Statement: Available at https://dx.doi. org/10.21037/tp-21-488 
Conflicts of Interest: All authors have completed the ICMJE uniform disclosure form (available at https://dx.doi. org/10.21037/tp-21-488). JY reports funding from the Education Department Science and Technology Research Project of Jiangxi Provincial (Youth Project) (190821). The other authors have no conflicts of interest to declare.

Etbical Statement: The authors are accountable for all aspects of the work in ensuring that questions related to the accuracy or integrity of any part of the work are appropriately investigated and resolved. The study was conducted in accordance with the Declaration of Helsinki (as revised in 2013). The study was approved by Scientific Research Ethics Committee of the First Affiliated Hospital of Gannan Medical College (No. LLSC-202105401) and informed consent was taken from all the patients' guardians.

Open Access Statement: This is an Open Access article distributed in accordance with the Creative Commons Attribution-NonCommercial-NoDerivs 4.0 International License (CC BY-NC-ND 4.0), which permits the noncommercial replication and distribution of the article with the strict proviso that no changes or edits are made and the original work is properly cited (including links to both the formal publication through the relevant DOI and the license). See: https://creativecommons.org/licenses/by-nc-nd/4.0/.

\section{References}

1. Demir IH, Erdem A, Sarıtaş T, et al. Diagnosis, treatment and outcomes of patients with aortopulmonary window. Balkan Med J 2013;30:191-6.

2. Tongprasert F, Sittiwangkul R, Jatavan P, et al. Prenatal Diagnosis of Aortopulmonary Window: A Case Series and Literature Review. J Ultrasound Med 2017;36:1733-8.

3. Louis-Jacques AF, Običan SG, Nguyen T, et al. Prenatal diagnosis of aortopulmonary window associated with

Cite this article as: $\mathrm{Yu} \mathrm{J}$, Liu $\mathrm{K}, \mathrm{Xu} \mathrm{W}$, Xiong J, Zhong X, $\mathrm{Hu} \mathrm{S}, \mathrm{Li}$ W. Prenatal ultrasound diagnosis and management of fetal aortopulmonary septal defects: a case series. Transl Pediatr 2021;10(11):3068-3074. doi: 10.21037/tp-21-488 aberrant subclavian artery. Cardiol Young 2017;27:1441-3.

4. Richardson JV, Doty DB, Rossi NP, et al. The spectrum of anomalies of aortopulmonary septation. J Thorac Cardiovasc Surg 1979;78:21-7.

5. Chen CH, Chen SJ, Qiu YW, et al. Prenatal ultrasonographic diagnosis of fetal aorticopulmonary septal defect. Journal of China Clinic Medical Imaging 2015;26:61-2.

6. Aslan H, Corbacioglu A, Yildirim G, et al. Prenatal diagnosis of aortopulmonary window. J Clin Ultrasound 2012;40:598-9.

7. Braunlin E, Peoples WM, Freedom RM, et al. Interruption of the aortic arch with aorticopulmonary septal defect. An anatomic review. Pediatr Cardiol 1982;3:329-35.

8. Luo CR, Gu XY, Wang SY, et al. Parental echocard iographic characterisctics of fetal aortopulmonary window. Chinese Journal of Medical Imaging Technology 2018;34:1848-51.

9. Lu Y, Liu F, Jia B, et al. Clinical analysis of 25 cases with aortopulmonary window. Chinese Journal of EvidenceBased Pediatrics 2013;8:167-71.

10. Bagtharia R, Trivedi KR, Burkhart HM, et al. Outcomes for patients with an aortopulmonary window, and the impact of associated cardiovascular lesions. Cardiol Young 2004; 14:473-80.

11. Ghelani SJ, Quinonez LG, Rathod RH. Prenatal Diagnosis and Management of Berry Syndrome, a Rare Conotruncal Anatomy. Circulation 2015;132:1593-4.

12. Fotaki A, Novaes J, Jicinska H, et al. Fetal aortopulmonary window: case series and review of the literature. Ultrasound Obstet Gynecol 2017;49:533-9.

13. Remon JI, Briston DA, Stern KW. Berry syndrome: the importance of genetic evaluation before surgical intervention. Cardiol Young 2016;26:188-90.

(English Language Editor: J. Teoh) 